\title{
DISSIPATION AND FORMATION OF GALACTIC HALOS
}

\author{
George R. Blumenthal \\ Lick Observatory, Board of Studies in Astronomy and Astrophysics \\ University of California, Santa Cruz, Santa Cruz, CA 95060, U.S.A.
}

When protogalaxies collapse, the cooling and infall of what will become the visible galactic component affects the mass distribution of dissipationless dark matter particles which constitute the halo. For spiral galaxies, the reaction of the dissipationless halo can have a striking effect on the resulting rotation curves [1-5].

The adiabatic invariant $\oint p d q$, where $q$ is a periodic coordinate and $p$ is its conjugate momentum, provides a convenient analytical tool for studying this phenomenon $[1-4,6]$ and has been extensively checked numerically $[1,5,7]$. For particles in circular orbits (which have the most phase space), the adiabatic invariant is $r M(r)$ provided that $M(r)$, the mass inside the orbital radius $r$, changes slowly in an orbital time.

Consider a spherically symmetric protogalaxy with a fraction $F \ll 1$ of dissipational baryons well mixed initially with a fraction $1-F$ of dissipationless particles. Define a truncation radius $R$ beyond which no dissipation occurs. The initial angular momentum of the protogalaxy is assumed to be small so that the dimensionless quantity $\lambda \equiv J|E|^{1 / 2} /\left(G \mathcal{M}^{5 / 2}\right) \ll 1$, where $\mathcal{M}$ is the total mass of a protogalaxy with total energy $E$. Numerical simulations show that tidal torques lead to a mean $\langle\lambda\rangle \approx 0.07$ with a width for the distribution $\Delta \lambda \approx 0.03[8]$

As the baryons dissipatively cool, fall in, and form a disk, a dark matter particle initially at radius $r_{i}$ will move in to radius $r<r_{i}$. The adiabatic invariant then implies that $r\left[M_{\text {disk }}(r)+\right.$ $\left.M_{\text {halo }}(r)\right]=r_{i} M_{i}\left(r_{i}\right)$, where $M_{i}\left(r_{i}\right)$ is the initial total mass distribution, $M_{\text {disk }}(r)$ is the final mass distribution of dissipational baryons, and $M_{\text {halo }}(r)$ is the final distribution of dissipationless halo particles. If the orbits of the halo particles do not cross, then $M_{\text {halo }}(r)=(1-F) M_{i}\left(r_{i}\right)$, which can be used to calculate the final radial distribution of the halo particles once $M_{i}\left(r_{i}\right)$ and $M_{\text {disk }}(r)$ are given. If the dissipational mass fraction $F \ll 1$, then the mass interior to a halo particle not too near the center of the protogalaxy undergoes a small fractional change in one orbital period, and the adiabatic invariant is expected to be a good approximation.

The initial protogalaxy is assumed to relax to an isothermal sphere. Large values of the core radius, $r_{\text {core }} / R \sim 0.4$, are needed for baryonic infall to produce observed spiral rotation curves [1]. Indeed, recent $\mathrm{N}$ body simulations $[9,10]$ show that $r_{\text {core }} / R \sim 0.2-0.5$ for cold dark matter spectra. The final mass distribution of the disk is assumed to be that of a thin disk of mass $\mathcal{M}_{\text {disk }}$ whose surface density $\mu_{\text {disk }}(r)=\mathcal{M}_{\text {disk }} \exp (-r / b) /\left(2 \pi b^{2}\right)$ decreases exponentially with scale length $b$.

The adiabatic approximation must break down near the center, where dissipating particles dominate the mass. However, $\mathrm{N}$-body simulations confirm that the total $M(r)$, and therefore the rotational velocity is quite well described by the adiabatic approximation for this case [7]. 
Fall and Efstathiou [11] first calculated the relation between the disk scale length and the initial angular momentum $\lambda$ of a protogalaxy containing both dissipational and dissipationless material. They assumed no transfer of angular momentum between the disk and halo components and no reaction of the halo to dissipative infall. We can relax the latter assumption $[1,2]$. If the dissipational material settles to an exponential disk, then the angular momentum of the thin disk is given by

$$
J_{\text {disk }}=2 \pi \int_{0}^{\infty} r d r \mu_{\text {disk }}(r) v_{\text {rot }}(r)
$$

where the rotation velocity $v_{\text {rot }}^{2}(r)=r\left[g_{\text {disk }}(r)+g_{\text {halo }}(r)\right]$. If there is no transfer of angular momentum between disk and halo particles, then $J_{\text {disk }} / \mathcal{M}_{\text {disk }}=J_{\text {halo }} / \mathcal{M}_{\text {halo, }}$, where $J_{\text {halo }}$ and $\mathcal{M}_{\text {halo }}$ are the halo angular momentum and mass out to the maximum radius of infall $R$.

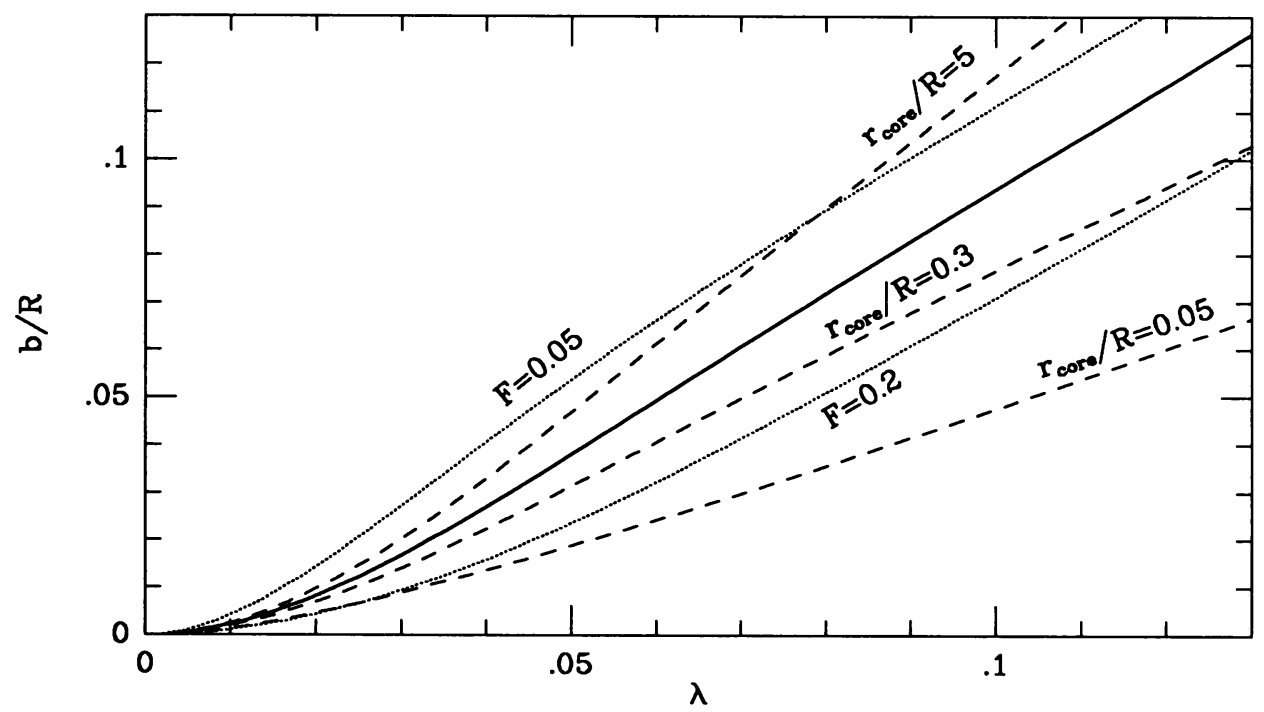

Figure 1: Plot of $b / R$ versus $\lambda$. The solid line has $F=0.1$ and $r_{\text {core }} / R=0.5$. The other curves are labelled with the one parameter which differs from these.

Figure 1 shows how the amount of dissipative infall $b / R$ is related to the initial angular momentum, $\lambda$. Smaller values of $F$ lead to less infall for a given $b / R$ therefore less disk angular momentum per unit disk mass. When $F \rightarrow 0$ the curve is expected to approach a limiting relation. Similarly, larger core radii produce less infall because there is less contribution of the halo to the disk rotation velocity and angular momentum. As $r_{\text {core }}$ becomes large, the initial protogalaxy density is nearly constant for $r<R$, and the curves become indistinguishable. A stiff halo which does not respond to the dissipative infall would lead to a larger value for the disk scale length $b$, about $27 \%$ larger for $\lambda=0.07$ and $F=0.1$ [2].

What circumstances will lead to flat rotation curves for spirals? One measure of flatness is the relative slope between 3 and 4.5 disk scale lengths $\left[v_{\text {rot }}(4.5 b)-v_{\text {rot }}(3 b)\right] / v_{\text {rot }}(4.5 b)$. Figure 2 shows a plot of this relative slope as a function of $\lambda$ and of $r_{\text {core }} / R$. Flat rotation curves arise for $r_{\text {core }} / R \geq 0.2$ and for $0.03 \leqslant \lambda \leqslant 0.1$ [1-3], which is just the region of parameter space relevant for cold dark matter [13] 


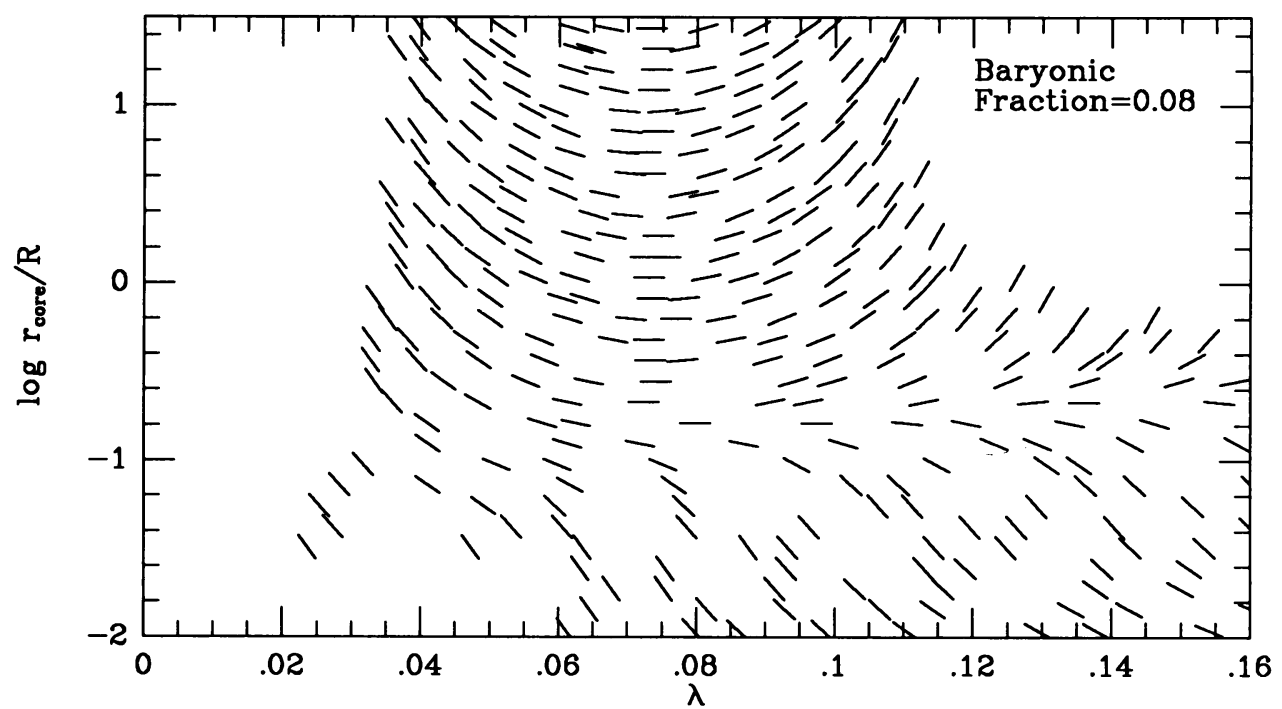

Figure 2: Relative slope between $r=3 b$ and $r=4.5 b$. The slopes are normalized so that a vertical line corresponds to the rotation curve of a pure disk. Observations suggest that real galaxies have slopes $<45$ degrees [12].

The observed rotation curves of spiral galaxies have been separated into three different classes which correlate much more strongly with environment than with Hubble type [15]. It is therefore of interest to calculate theoretical rotation curves to set limits on the parameters consistent with relatively flat rotation curves out to large distances and to determine which parameters may be responsible for the various forms of rotation curves which are observed [2].

Figure 3 shows the rotation velocity versus radial distance measured in units of the scale length, $r / b$. Again, flat rotation curves arise for $.05<F<0.1, r_{\text {core }} / R z .2$, and $.04<\lambda<0.1$. When these parameters are varied, both rising and falling rotation curves result beyond a few disk scale lengths.

In Figure 3 no feature in the rotation profile separates the inner, disk-dominated region from the outer, halo-dominated region [1-3]. The coupled motion of the two components, as the baryons fall into the center, avoids any noticeable separation of the two components. This is a direct consequence of the response of the halo to the infall of the visible matter. The absence of such a separation into two components has been noted observationally $[12,14-16]$.

What is responsible for the various forms of rotation curves seen in spiral galaxies? An obvious candidate is the bulge-to-disk ratio, but no correlation is observed between the form of the rotation curve and either Hubble type or bulge-to-disk ratio [15]. However, Figure 3 suggests that variations in the initial angular momentum $\lambda$ or in the core radius $r_{\text {core }} / R$ from galaxy to galaxy may lead to various forms for the mass distribution, assuming that $F$ is a universal constant. Figure 3 suggests that galaxies with a higher value of $\lambda$ will show a more steadily rising rotation curve. If field spirals have systematically lower initial angular momenta $\lambda$ than spirals in clusters, then one would expect field spirals to show more steadily rising rotation curves than cluster spirals, as has been observed [15].

Finally, earlier work [11] has suggested that the relation between $\lambda$ and $b / R$ is indeed quite 


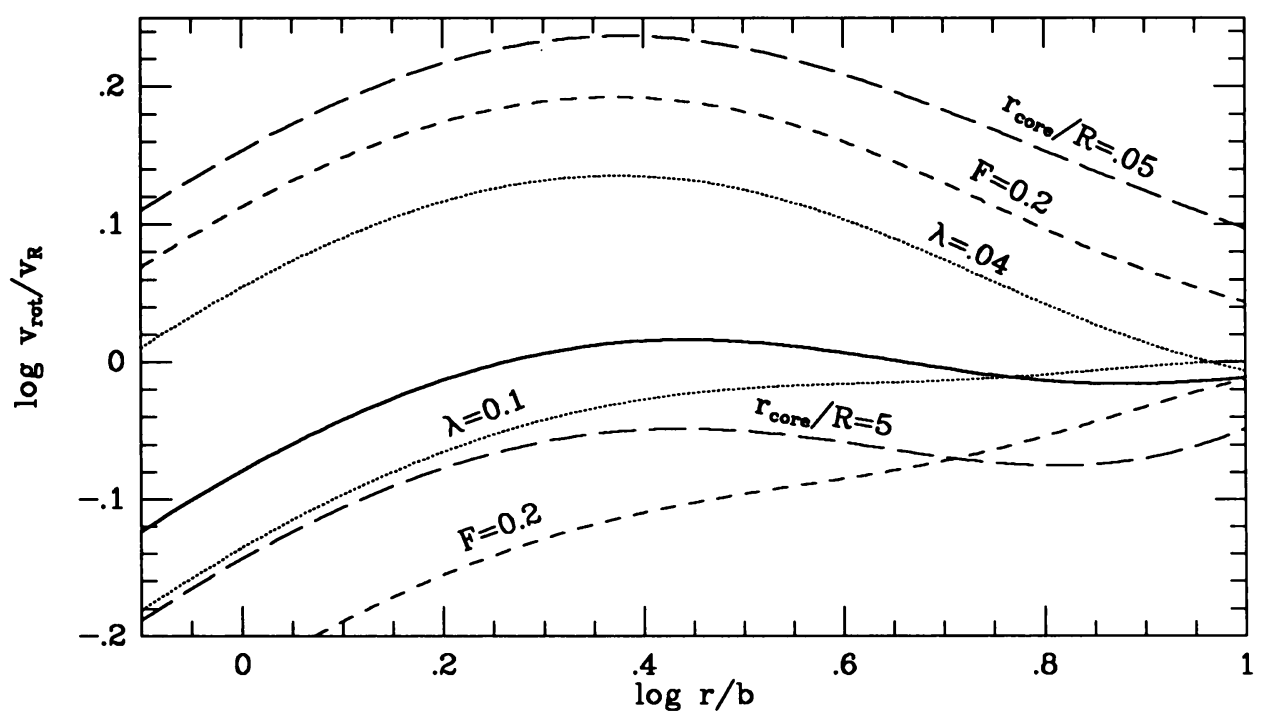

Figure 3: Rotation curves $\log v_{\text {rot }} / v_{R}$ versus $\log r / b$. The solid line corresponds to $\lambda=.07, F=0.1$, and $r_{\text {core }} / R=0.5$. The other curves are labelled with the one parameter which differs from these values.

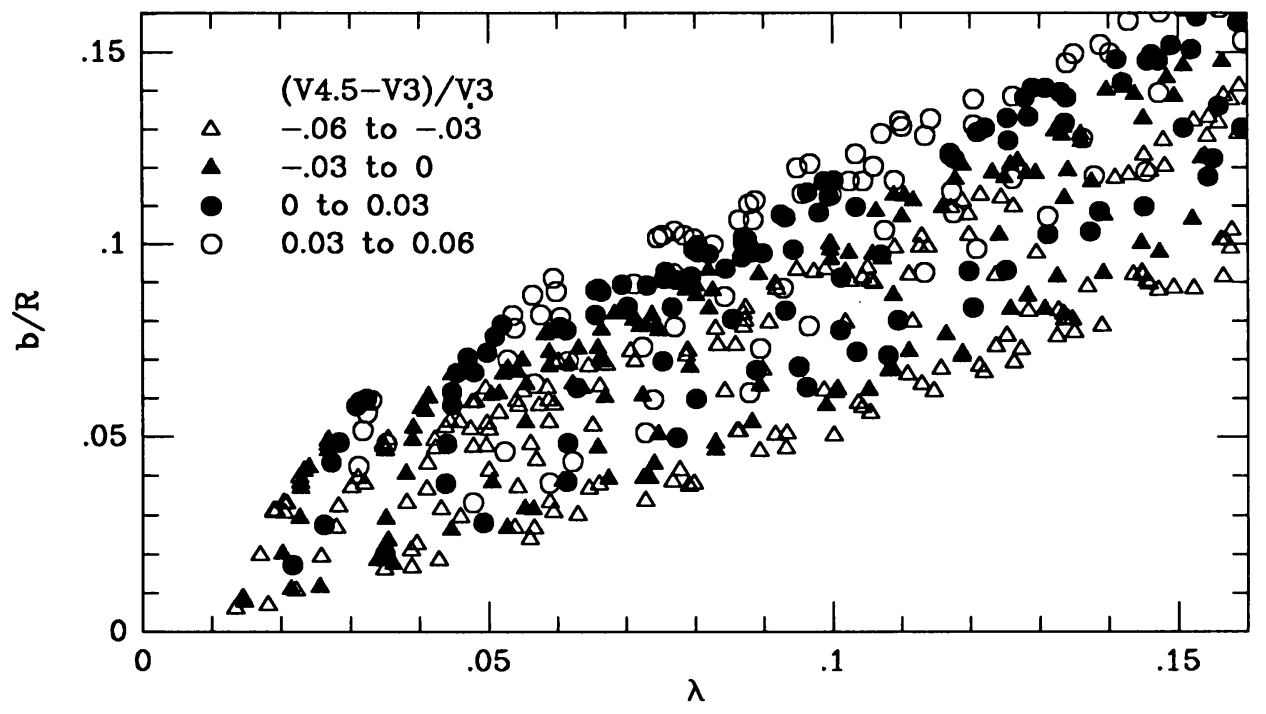

Figure 4: Plot of $\lambda$ versus $b / R$ for models with $v_{\max }\left(b / \mathcal{M}_{\text {disk }}\right)^{1 / 2}$ between 0.65 and 1.8 . 
narrow for real galaxies. This is tested in Figure 4, which plots the relation for those models with $v_{\max }\left(b / \mathcal{M}_{\text {disk }}\right)^{1 / 2}$ between 0.65 and 1.8 and with $|v(4.5 b)-v(3 b)| / v(3 b)<0.06$. The first criterion comes from [11], and the second from observed radio rotation curves. The result is a rather broad relation spanning about a factor of 2 in $\lambda$. This occurs because of the larger range of rotation curve slope observed than is described by the parameterization of [11]. Hence, an observed scale length predicts the original $\lambda$ to no better than a factor of two.

I gratefully acknowledge many stimulating interactions with my colleagues Ricardo Flores, Sandra Faber, and Joel Primack, with whom much of the work reported here was done. This research was supported by NSF grant PHY84-15444 and by a faculty research grant at U.C.S.C.

\section{$\underline{\text { References }}$}

1. G. R. Blumenthal, S. M. Faber, R. Flores and J. R. Primack, Ap. J. 301, 27 (1986)

2. G. R. Blumenthal, R. Flores, S. M. Faber and J. Primack, in preparation (1987)

3. G. R. Blumenthal, in Nearly Normal Galaxies, ed. S. Faber (Springer, New York), 1987, p. 401.

4. B. Ryden and J. Gunn, Bull. AAS 16, 487 (1984)

5. J. Barnes, in Nearly Normal Galaxies, ed. S. Faber (Springer, New York), 1987, p. 154.

6. Ya. B. Zeldovich, A. Klypin, M. Yu. Khlopov and V. Chechetkin, Sov. J. Nucl. Phys., 31, 664 (1980)

7. K. S. Oh, in preparation (1987)

8. G. Efstathiou and B. J. T. Jones, M.N.R.A.S. 186, 133 (1979)

9. C. Frenk, S. White, G. Efstathiou and M. Davis, Nature 317, 595 (1985)

10. P. Quinn, J. Salmon, and W. Zurek, preprint (1986)

11. S. M. Fall and G. Efstathiou, M.N.R.A.S. 193, 189 (1980)

12. T. S. van Albada and R. Sanchisi, preprint (1986)

13. G. Blumenthal, S. Faber, J. Primack, and M. Rees, Nature 311, 517 (1984); also Nature 313, 72 (1985)

14. D. Burstein and V. Rubin, Ap. J. 297, 423 (1985)

15. D. Burstein, V. Rubin, W. Ford and B. Whitmore Ap. J. Letters 305, L11 (1986)

16. J. Bahcall and S. Casertano, Ap. J. Letters 293, L7 (1985) 